\title{
Enlightenments of Female Roles Singing in Jingzhou Huagu Opera to the National Vocal Music Teaching
}

\author{
LI Jun \\ Art college ,Jingchu University of Technology,Jingmen 448000,China \\ lijunjc@126.com
}

\begin{abstract}
Keywords: Huagu opera; Female role; National vocal music teaching
Abstract. In the traditional culture system our country, the traditional opera art is the essence of national vocal music art. It is also the crystallization of the wisdom of the hard-working people. From the point of our national vocal music art form, its connotation mainly consists of "gas, word, cavity, feeling and taste" five aspects, for the national vocal music art research also focus on the five aspects. Jingzhou opera as a kind of local opera, it is the important part of the southern ethnic vocal music art, whose singing has a unique charm, especially the woman's role, the role includes more factors of national vocal music teaching, thus has very high reference value for the national vocal music teaching.
\end{abstract}

\section{Introduction}

National vocal music teaching has a history of one thousand years in China; it is a kind of art form that based on the national language, after the evolution of the long-term development which has been to the agreement and China's vocal music aesthetic. Due to the different geographical environment, the humanities in the national vocal music art south and north areas in China have big difference. As an important role in the stage of opera performance, so to speak, the woman's role focus on a variety of singing techniques and main points, has a unique charm, stage performance and Jingzhou opera is one of the important types in the south. The local characteristics of it is very clear, the audience is very extensive. In the current national vocal music teaching, the positive reference and the opera stage performance skills is of great significance, it can help students understand and grasp the national vocal music art connotation better, thus make the teaching more scientific and efficient.

\section{Sing according to the words principle in stage performance}

National vocal music art is all about the combination of national culture to embody national characteristic through vocal music, and this characteristic also has close relationship with regional culture. From the point of view, singing style of any kind of opera stage, in the process of vocal music teaching, students need to be fit the actual, to carry out targeted teaching according to the difference between the students. [1] From a practical perspective, it can be divided into four different stages, which are the primary stage, intermediate stage and advanced stage and research stage. The four stages of teaching each have their own characteristics and laws. First, in the primary stage, the teacher must set up the reasonable learning objectives and tasks according to the student's understanding ability to decrease the difficulty of learning the target can be simply described as follows: in the pronunciation of the middle and lower acoustic area to guide correctly, students gradually form the low bits, the shape of standard laryngeal pharynx, and his breathing, in pronunciation quality is basically in a state of relaxation and round, but not beyond the interval of pure 8 degrees, and also to keep smooth connection and unity. In addition, also to ensure that can effortlessly sang some elementary level in pronunciation, and on the track contains connotation, mood and style to be able to understand and grasp correctly; [2]Secondly, for the intermediate stage and advanced stage of vocal music teaching, teachers should also be suitable according to the student ability of this stage of the teaching objectives and tasks, which is on the basis of the basic singing skills, can the common state of singing and points are more skilled master, for example, in the form of laryngeal pharynx to correctly understand the correct adjustment and exhale and inhale voice and breath balance 
processing, and so on, after singing master these points can be stable interval within 10 degrees of songs, and keep the notes right connections, can also form a bass tone in the area, and skilled treble volume strength control ability, after the basic ability to achieve, can also according to the types and features of the singing songs right clearly express the music rhyme beauty, make different styles of language processing with ease, under the premise can play different tracks in the woman's role, to the singing the development level of different tracks will also be able to correct understanding and expression, so that the music emotion and connotation of more rich, more can cause the audience resonance in one's mind[3].

What talked above is the national vocal music teaching in pronunciation skills requirement, but due to the national vocal music contains a strong regional culture characteristics, it also cannot leave the response to the national style, stage performance in the stage performance to correctly grasp the national style, you need to use the regional cultural features and national folk music works to reflect, only in this way can increase the richness of national vocal music performance and regionality. For the primary stage of vocal music teaching, in the process of teaching repertoire can't too much about this, and sing also cannot too difficult, so it is easy to damage to established laryngopharyngeal form. For example Jingzhou area of national vocal music "double left bamboo", "little son", and so on all can be used as the primary stage and middle stage repertoire of vocal music teaching. The two songs is the representative of the national vocal music jingzhou area, according to the two pieces of music, its tone is more moderate, pinyin combination between more closely, the singer in the singing is active when the mood, anacreontic, shaped by the character is not complicated, and explains the contents of a whole repertoire is a pair of girls to go out to do farm work in the morning happy state of mind (where the farm work is playing a ragweed). For the primary stage and middle stage of vocal music students, can be more accurate experience and show the character of psychological change, and master the contained style and lasting appeal, teaching value is very high. According to students' understanding situation can continue with falling tone singing style is given priority to, the song singing opera language mainly embodies the Jingzhou today the paper dialect, the characteristics of the singing language spoken is obvious, the combination between word cavity and tone is very tight, therefore, for students, there will be no greater difficulties in learning, day only needs to master the basic tone of the paper dialect and pronunciation characteristics, in the process, cooperate with the teacher's demonstration can be skilled in mind, with ease, also can be more than listen to the recording data, can be very natural pronunciation and singing.[4] Conversely, if the primary stage of students in learning, according to the score only when breaks away from practice will have great difficulty, the tone is also difficult to accurately grasp, prone to walk out of tune sound phenomena, such as singing the word "mountain" cultured embellish cavity, the word "to" sing for error phenomenon such as "ke" by mistake.

From the view of the teachers of national vocal music teaching, vocal music teachers can sing opera stage the role according to Jingzhou characteristics and students' actual use local materials, setting up reasonable teaching content and teaching program, through the opera singing songs, guides the student to contact Jingzhou and practice to understand and grasp the importance of "improperness in accordance with the word" purpose of a cover style place in better performance. In addition, it still have a lot to do with the current national music form of his works, many current music creator created music works are based on the local culture and national features and creation, to truly express the connotation of songs, the singer must deeply grasp national language features, has profound understanding to the regional culture, to grasp the national song singing style. In addition, in the national vocal music teaching practice, vocal music teachers need to let students understand the importance of national singing songs in the enunciation to rhyme and through various ways. From singing, articulation is one of the most basic requirements, must want to standard pronunciation, and this also is general international, such as Italy regional music not only emphasize on the singing clear articulation standard, but also pay attention to vowel and continue.[5] Our Chinese language is evolved after one thousand years development, has the characteristics of speech complexity and emphasizes diversity, after years of research can be simply summarized the language pronunciation characteristics, namely the words "strong", the word for a "mouth", after carefully experience "'" 
heavy, slow, abdominal tail light" the difference between a and so on. Study for students of ethnic vocal music, you need to Chinese pronunciation skills and rules of a correct understanding, and their own pronunciation errors to correct, such ability can do well in the stage performance, the audience can also clearly understand the content of the songs, to more accurately understand the works contained in the content, form and the psychological resonance of performers.

\section{Function analysis of the spoken parts on the stage}

Dialuoging in China occupies an extremely important role in Chinese opera singing, which is the key of singing effect is guaranteed. From the importance of the white point of view, it is essentially a comprehensive "namely", "sound", "improperness" and "air" as one of the system, for the singer, as long as white good practice, we can adjust their singing very well state, while singing vocal cords and reasonable utilization, correct control of breath rhythm and scientific regulation of resonance can be skilled to complete, and on this basis can also be dripping wet show the tracks in the feelings of the characters, the whole stage of flesh and blood, this paper analyzes the Jingzhou hu the white also has the function of woman's role.

In the national vocal music teaching, the students at the intermediate stage and advanced stage is bound to learn about the national opera singing skills and requirements. In the actual teaching process, teachers will find a problem, is the student's pronunciation skills to master is very good, but one's own emotions and inner activities also are far apart with the characters, performance is relatively rigid, for solving this problem, teachers can guide students to learn other relatively simple opera white, also can be actual, several section of the songs to correctly adjust itself in practice, close to the characters of more accurate. [6]In addition, in the national vocal music in actual teaching, there is a problem, is that the student is national music style and lasting appeal of understanding and mastering is given priority to with pronunciation training, and this way of training is not enough, but if in the case of foundation weak students emphasize the singing style will cause a drop in the quality of pronunciation, it is difficult to achieve the expected teaching effect. From the perspective of art, art performance and pronunciation skills are irreconcilable contradictions existing in vocal music teaching, if the solution is not good, pronunciation skills is hard to fully reflect; If solve well, both can form the effect of supplement each other and promote each other, the vocal music performance is very good. Accordingly in the intermediate stage of the vocal music teaching, the teacher can't completely according to the advanced teaching mode to teaching, students must fit the actual, targeted, planned for the establishment of teaching plan, only this can highlight the charm of national vocal music art, let students experience the connotation of national vocal music art, learning more effective.

\section{Lubrication}

Jingzhou Huagu opera as local operas, its sing is of strong national characteristics. From the aspects of singing, embellish cavity in the actual singing method basically has the following kinds: first, Jingzhou opera embellish chamber mainly reflect the amorous feelings of the ethos of Jianghan plain and the aesthetic habits of the masses, very strong regional characteristics, national style is more protruding. Words in the song singing cavity effect is to add flower decoration, from the perspective of the produce of it, it is on the basis of the original singing, derived by different tone combination of Chinese characters and the cavity (the word is the word and the word or words between the tails of a small cavity) has unique characteristics. For example in the rush palace drama madam chun heung-lin a singing, word cavity of these features is the ability to guarantee basic singing more plump and smooth melody, could be more apt to show the characters of the emotion change; Second, the sound. Shortness of the sound is a kind of lip-synching skills, tone up or to decline; Third, vibrato, Jingzhou opera singing is the tremolo is mainly in the long short vibrato and tremolo two kinds, the former is above the tremolo is given priority to, the latter is below the tremolo is given priority to; Fourth, cavity, the cavity method mainly is end to fall, for example in the western chamber, the wind blowing 
willow all line plays its singing at the end of each sentence there will be a gradual decline in the state, but it is not the same as other types of cavity.

The above several embellish cavity method is singing opera stage the role of Jingzhou area unique singing skills, in addition to these aspects, it also involves several other singing skills and methods, such as "appoggiatura", "" mad" and so on. In the current national vocal music teaching, the teacher to the singing skills to explain to the students the right way, the students in mastering these unique embellish cavity method, can in the singing style of living museum or is a living museum of play, can play against three singing effect at a stroke, so as to achieve with ease, skilled in the effect of the heart. In singing of departure at the fron program, for example, "WeiCheng toward the light rain city dust, guest qingqing LiuSe new" word, basically every word contains the appoggiatura decoration, like "guest" word contains a tremolo cavity skills, these techniques for lip cavity occupies an important position in the whole play, it is the key to get the best results play singing, once the lack of the cavity skills, play singing will lose the unique flavor of the guqin effect of the whole process of performance and performance will seem plain, there is no appeal.

\section{Summary}

As one of the important types of southern region, Jingzhou opera singing play contains full-bodied local characteristics, which is a concentrated reflection of regional culture. Use the performing opera stage the role of the techniques and methods in the national vocal music teaching can make the students understand of the connotation of national vocal music and emotion more profoundly and accurately, thus realize the Jingzhou opera unique charm. In the actual teaching process, teachers should grasp the role's woman's basic skills according to the actual students, and use the right and reasonable teaching method to make students form a system of national vocal music study and complete system, so as to achieve the expected teaching effect, to promote the innovation and development of Jingzhou opera performances,

\section{References}

[1] Xu Liya, Jiang Wenjuan jiang. Enlightenments of Jingzhou opera stage singing to the teaching of national vocal music [J]. Journal of Hubei institute of adult education, 2015 (5) : 88-89.

[2] He Yiming. Opera training mode in the national vocal music teaching the application [J]. Journal of art, 2013, (10): 72-73.

[3] Tan Yihong. Opera into the vocal music teaching of high school study [J]. Journal of drama house, 2015, (7): 243-244.

[4] Ceng Xiangling, Ceng Xiangsi. His era of hunan opera footprint - from "bang cut firewood" reform caused by intravenous drip thinking [J]. Journal of art, 2014, (2): 49-52.

[5] Wujing. Look from the material change jingzhou opera artistic features of the [J]. Journal of hubei academy of social sciences, 2015, (1): 194-198.

[6] Zhang Mi. Try to talk about the national vocal music in hunan opera of the reference [J]. Journal of popular songs, 2014 (5): 79-80. 